\title{
A STRUT FINITE ELEMENT FOR EXACT INCOMPRESSIBLE ISOTROPIC HYPERELASTIC ANALYSIS
}

Vinicius F. ARCARO*1, Pietro C. FERRAZZO'

\section{Abstract}

This text describes a mathematical model of a strut finite element for isotropic incompressible hyperelastic materials. The invariants of the Right Cauchy-Green deformation tensor are written in terms of nodal displacements. The equilibrium problem is formulated as an unconstrained nonlinear programming problem, where the objective function is the total potential energy of the structure and the nodal displacements are the unknowns. The constraint for incompressibility is satisfied exactly, thereby eliminating the need for a penalty function. The results of the examples calculated by the proposed mathematical model show five significant digits in agreement when compared with commercial finite element analysis software.
Address

1 College of Civil Engineering University of Campinas, Campinas, Brazil

* Corresponding author: vinicius.arcaro@gmail.com

Key words

- Hyperelasticity,

- Incompressibility,

- Minimization

- Nonlinear

- Finite element.

\section{INTRODUCTION}

This text describes a mathematical model of a strut finite element for isotropic incompressible hyperelastic materials. The deformation gradient tensor is written in terms of nodal displacements. The invariants of the Right Cauchy-Green deformation tensor are written in terms of nodal displacements. The constraint for incompressibility is satisfied exactly, thereby eliminating the need for a penalty function. The equilibrium problem is formulated as an unconstrained nonlinear programming problem, where the objective function is the total potential energy of the structure and the nodal displacements are the unknowns. The total potential energy is minimized using a quasi-Newton method.

The total potential energy minimization method for the analysis of cable structures was first described by (Buchholdt, 1966). The minimization of the total potential energy to find an equilibrium for cable network analysis was used by (Coyette and Guisset, 1988), where the authors noticed that a better solution could be achieved by resorting to the basic energy concepts underlying the stiffness approach. The strain energy density function (strain energy per unit of undeformed volume) representing incompressible hyperelastic materials usually incorporates a penalty function to account for the incompressibility. The aug- mented Lagrange method was proposed by (Brinkhues et al., 2013) to avoid numerical difficulties arising from the use of a penalty parameter. A review of the augmented Lagrangian and penalty methods that have been successfully employed to enforce incompressibility, which focus on cardiac mechanics, was presented by (Hadjicharalambous et al., 2014). In the literature, a nearly incompressible material is used to denote a material that is incompressible, but its numerical treatment involves a small volumetric deformation. The importance of a better way to tackle incompressibility relies on the fact that incompressible hyperelastic material models have been widely used for modelling the mechanical behaviour of soft biological tissues. The work of (Mustafy et al., 2014) noticed that articular ligaments were mostly modeled with strut elements. A detailed review of Isotropic incompressible hyperelastic material models used for modelling soft biological tissues was presented by (Wex et al., 2015).

\section{NOTATION}

The following notation is used for vector and tensor algebra: A Greek letter represents a scalar. A lower case letter represents a point or a column vector in a three dimensional Cartesian space. An upper 
case letter represents a matrix or a tensor. For clarity, the summation convention will not be used in this text.

\section{STRUT ELEMENT DEFINITION}

Figure 1 shows the geometry of the strut element in a three dimensional Cartesian space. The nodes are labeled 1 and 2. The nodal displacements transform the element from its undeformed or initial state to its deformed or final state. Notice that the four vectors may be non-coplanar vectors. Since the vectors form a closed loop, the vector sum of all four vectors must be zero. The vectors $u$ and $\bar{u}$ are unit vectors in the direction of the element in the initial and final state, respectively. The scalars $\lambda$ and $\bar{\lambda}$ are the lengths of the element in the initial and final state, respectively. The nodal displacements $d^{1}$ and $d^{2}$ move points $x^{1}$ and $x^{2}$ in the initial state to points $\bar{x}^{1}$ and $\bar{x}^{2}$ in the final state, respectively.

The relationship between the vectors describing the strut element

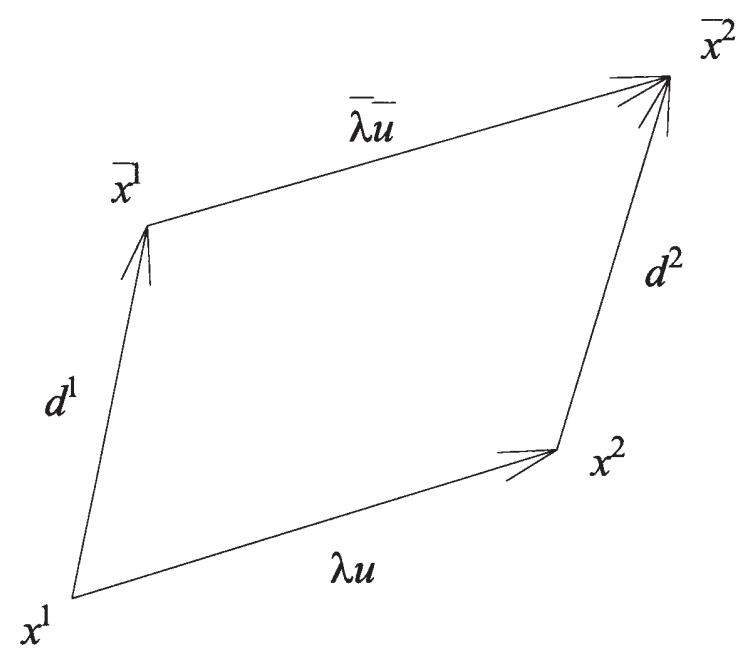

Fig. 1 Strut Element

geometry can be written as follows:

$$
\lambda u+d^{2}-\bar{\lambda} \bar{u}-d^{1}=0
$$

Letting,

$$
z=\left(d^{2}-d^{1}\right) / \lambda
$$

The relationship between the vectors describing the strut element geometry becomes:

$$
\bar{\lambda} \bar{u}=\lambda u+\lambda z
$$

\subsection{Convex Combination of Vertexes}

The convex combination of vertexes in the deformed state is given by:

$$
\begin{gathered}
\bar{x}=\alpha_{1} \bar{x}^{1}+\alpha_{2} \bar{x}^{2} \\
\alpha_{1}+\alpha_{2}=1 \mid \alpha_{i} \geq 0
\end{gathered}
$$

Considering that a point in the strut element can be described by the same convex combination of vertexes in the undeformed and deformed states, a point in the deformed state can be written as:

$$
\bar{x}=\alpha_{1} d^{1}+\alpha_{2} d^{2}+x
$$

\subsection{Alpha Coefficients}

The alpha coefficients can be determined through the convex combination of vertexes in the undeformed state as:

$$
\begin{aligned}
& \alpha_{1}=u^{T}\left(x^{2}-x\right) / \lambda \\
& \alpha_{2}=u^{T}\left(x-x^{1}\right) / \lambda
\end{aligned}
$$

\subsection{Geometric Interpretation}

Each alpha coefficient corresponds to a division of a fraction of the undeformed length by the total undeformed length. Figure 2 shows point $x$ as a convex combination of vertexes in the undeformed state.

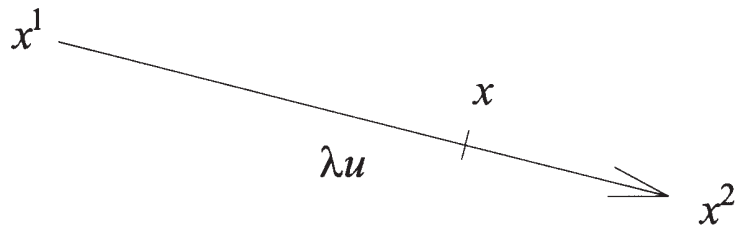

Fig. 2 Convex combination of vertexes in the undeformed state

$$
\begin{gathered}
\left(x^{2}-x\right)=\left\|x^{2}-x\right\| u \\
\alpha_{1}=\left\|x^{2}-x\right\| / \lambda
\end{gathered}
$$

Notice that the non-negative constraint for $\alpha_{1}$ is satisfied. A corresponding expression can be written for $\alpha_{2}$.

\subsection{Severe Cancellation}

Inaccuracy often results from severe cancellation that occurs when nearly equal values are subtracted as described by (Goldberg, 1991). Severe cancellation can usually be eliminated by algebraic reformulation. Notice that inaccuracy can result from expressions involving the difference between the deformed length and the undeformed length because this difference can be arbitrarily small. This difference should be written as:

$$
\bar{\lambda}-\lambda=\lambda\left(2 u^{T} z+z^{T} z\right) /\left[\left(1+2 u^{T} z+z^{T} z\right)^{1 / 2}+1\right]
$$

To avoid severe cancellation, every expression should be checked and eventually reformulated in order to be used in a computer code.

\section{DEFORMATION GRADIENT TENSOR}

The convex combination of vertexes in the deformed state can be written as:

$$
\bar{x}=x+u^{T} x z+d^{2}-u^{T} x^{2} z
$$

This convex combination of vertexes is a deformation mapping that transforms points from the undeformed state to the deformed state. The derivatives of the deformation mapping can be written as:

$$
\frac{\partial \bar{x}_{i}}{\partial x_{j}}=\delta_{i j}+z_{i} u_{j}
$$

The components of the deformation gradient tensor $F$ can be written as: 


$$
f_{i j}=\frac{\partial \bar{x}_{i}}{\partial x_{j}}=\delta_{i j}+z_{i} u_{j}
$$

The deformation gradient tensor $F$ can be written as:

$$
F=I+z u^{T}
$$

However, the previous expression require additional terms to account for the deformation orthogonal to the element's line. These additional terms will satisfy the questions raised by (Kaklamanis and Spiliopoulos, 2007), which points that the effect of the change in the cross sectional area of the strut element during its motion is not usually formalized on theoretical grounds.

Consider unit vectors $v$ and $w$, which are orthogonal to $u$ and unit vectors $\bar{v}$ and $\bar{w}$, which are orthogonal to $\bar{u}$, such that:

$$
\begin{gathered}
w=u \times v \\
\bar{w}=\bar{u} \times \bar{v}
\end{gathered}
$$

The additional terms to account for the deformations orthogonal to the element's line must vanish when the expression is applied to vector $u$. Considering $b$ and $c$ as vectors, one possible expression can be written as:

$$
F=I+z u^{T}+b v^{T}+c w^{T}
$$

Additionally, applying this expression to an undeformed length must result in the corresponding deformed length. In the direction parallel to the element's line:

$$
F(\lambda u)=\lambda u+\lambda z=\bar{\lambda} \bar{u}
$$

Consider the scalars $\delta$ and $\bar{\delta}$ equal to the thickness of the element in the undeformed state and deformed state respectively. Letting,

$$
\rho=\bar{\delta} / \delta
$$

In the $v$ direction orthogonal to the element's line:

$$
\begin{gathered}
F(\delta v)=\delta v+\delta b \\
F(\delta v)=\bar{\delta} \bar{v} \Rightarrow b=\rho \bar{v}-v
\end{gathered}
$$

In the $w$ direction orthogonal to the element's line:

$$
\begin{gathered}
F(\delta w)=\delta w+\delta c \\
F(\delta w)=\bar{\delta} \bar{w} \Rightarrow c=\rho \bar{w}-w
\end{gathered}
$$

Then, the deformation gradient tensor can be written as:

$$
F=I+z u^{T}+(\rho \bar{v}-v) v^{T}+(\rho \bar{w}-w) w^{T}
$$

The invariant 3 of $F$ can be interpreted as the deformed volume divided by the undeformed volume.

$$
f_{3}=\operatorname{det}(F)=\rho^{2}\left(1+2 u^{T} z+z^{T} z\right)^{1 / 2}
$$

\section{RIGHT CAUCHY-GREEN DEFORMATION TENSOR}

The right Cauchy-Green deformation tensor $C$ is given in terms of the deformation gradient tensor $F$ as:

$$
C=F^{T} F
$$

The invariants can be written as:

$$
\begin{gathered}
c_{1}=\operatorname{tr}(C)=1+2 u^{T} z+z^{T} z+2 \rho^{2} \\
c_{2}=\operatorname{tr}\left(C^{T} C\right)=\left(1+2 u^{T} z+z^{T} z\right)^{2}+2 \rho^{4} \\
c_{3}=\operatorname{det}(C)=\left(1+2 u^{T} z+z^{T} z\right) \rho^{4}
\end{gathered}
$$

\section{STRAIN ENERGY DENSITY FUNCTION}

Consider $\psi$ as the strain energy density function.

$$
\psi=\psi\left(c_{1}, c_{2}, c_{3}\right)
$$

The derivative of $\psi$ with respect to the invariant $i$ of $C$ is denoted by $\psi_{i}$ and can be written as:

$$
\psi_{i}=\frac{\partial \psi}{\partial c_{i}} \mid i=1,2,3
$$

Notice that the strain energy density function and its derivatives with respect to the invariants of the right Cauchy-Green deformation tensor depend on the material.

\section{TOTAL POTENTIAL ENERGY}

Consider $\alpha$ as the area of the element in the undeformed state and $\omega$ as the work done by external forces. The total potential energy $\phi$ can be written as a function of the unknown displacements by the summation of the strain energy over all elements minus the work done by the external forces:

$$
\phi=\sum_{\text {elements }} \psi \alpha \lambda-\omega
$$

The gradient of the total potential energy can be written as a function of the unknown displacements by the summation of the gradient of the strain energy function with respect to the nodal displacements over all the elements minus the gradient of the work done by the external forces with respect to the nodal displacements.

$$
\nabla \phi=\sum_{\text {elements }}(\nabla \psi) \alpha \lambda-\nabla \omega
$$

For each element, the gradient of the strain energy function with respect to the nodal displacements of the element can be calculated by using the chain rule as:

$$
\nabla \psi=\psi_{1} \nabla c_{1}+\psi_{2} \nabla c_{2}+\psi_{3} \nabla c_{3}
$$

\section{CAUCHY STRESS TENSOR}

According to (Bonet and Wood, 2008), for the case of isotropic hyperelasticity, the Cauchy stress tensor can be written as:

$$
S=\frac{2}{f_{3}} F \frac{\partial \psi}{\partial C} F^{T}
$$

However

$$
\begin{gathered}
\frac{\partial \psi}{\partial C}=\psi_{1} \frac{\partial c_{1}}{\partial C}+\psi_{2} \frac{\partial c_{2}}{\partial C}+\psi_{3} \frac{\partial c_{3}}{\partial C} \\
c_{1}=\operatorname{tr}(C) \Rightarrow \frac{\partial c_{1}}{\partial C}=I
\end{gathered}
$$




$$
\begin{aligned}
& c_{2}=\operatorname{tr}\left(C^{T} C\right) \Rightarrow \frac{\partial c_{2}}{\partial C}=2 C \\
& c_{3}=\operatorname{det}(C) \Rightarrow \frac{\partial c_{3}}{\partial C}=f_{3}^{2} C^{-1}
\end{aligned}
$$

Therefore,

$$
S=2 \psi_{1} / f_{3} F F^{T}+4 \psi_{2} / f_{3} F F^{T} F F^{T}+2 \psi_{3} f_{3} I
$$

\section{INCOMPRESSIBILITY}

A hydrostatic pressure can be applied to an incompressible solid without changing its shape. The strain energy density is a function of only two invariants, but it can be written in the following way to produce an unknown hydrostatic pressure in the Cauchy stress tensor. This unknown hydrostatic pressure will be determined by a boundary condition on the principal stress orthogonal to the element's line.

$$
\begin{gathered}
\psi=\psi\left(c_{1}, c_{2}\right)-\gamma / 2\left(c_{3}-1\right) \\
\frac{\partial \psi}{\partial C}=\psi_{1} \frac{\partial c_{1}}{\partial C}+\psi_{2} \frac{\partial c_{2}}{\partial C}-\gamma / 2 \frac{\partial c_{3}}{\partial C} \\
S=2 \psi_{1} F F^{T}+4 \psi_{2} F F^{T} F F^{T}-\gamma I
\end{gathered}
$$

\subsection{Thickness}

By setting invariant 3 of the deformation gradient tensor $F$ equal to 1 , the thickness of the element in the deformed state can be written as:

$$
f_{3}=1 \Rightarrow \rho^{2}=1 /\left(1+2 u^{T} z+z^{T} z\right)^{1 / 2}
$$

\subsection{Stress Orthogonal to the Element's Line}

The traction vector related to a unit vector orthogonal to the element's line in the deformed state can be written as:

$$
S \bar{v}=\left(2 \psi_{1} \rho^{2}+4 \psi_{2} \rho^{4}-\gamma\right) \bar{v}
$$

The unit vector $\bar{v}$ is a principal direction associated with the principal stress given by:

$$
\sigma=2 \psi_{1} \rho^{2}+4 \psi_{2} \rho^{4}-\gamma
$$

The boundary condition implies that this stress is equal to zero. Therefore, the Cauchy stress tensor can be written as:

$$
S=2 \psi_{1} F F^{T}+4 \psi_{2} F F^{T} F F^{T}-\left(2 \psi_{1} \rho^{2}+4 \psi_{2} \rho^{4}\right) I
$$

\subsection{Stress Parallel to the Element's Line}

The traction vector related to the unit vector parallel to the element's line in the deformed state can be written as:

$$
S \bar{u}=\left\{2 \psi_{1}\left[(\bar{\lambda} / \lambda)^{2}-\rho^{2}\right]+4 \psi_{2}\left[(\bar{\lambda} / \lambda)^{4}-\rho^{4}\right]\right\} \bar{u}
$$

The unit vector $\bar{u}$ is a principal direction associated with the principal stress given by:

$$
\sigma=2 \psi_{1}\left[(\bar{\lambda} / \lambda)^{2}-\rho^{2}\right]+4 \psi_{2}\left[(\bar{\lambda} / \lambda)^{4}-\rho^{4}\right]
$$

However,

$$
\begin{aligned}
& (\bar{\lambda} / \lambda)^{2}=1+2 u^{T} z+z^{T} z \\
& \rho^{2}=1 /\left(1+2 u^{T} z+z^{T} z\right)^{1 / 2}
\end{aligned}
$$

Therefore, the principal stress can be written as:

$$
\begin{aligned}
& \sigma= \\
& +2 \psi_{1}\left[\left(1+2 u^{T} z+z^{T} z\right)-1 /\left(1+2 u^{T} z+z^{T} z\right)^{1 / 2}\right]+ \\
& +4 \psi_{2}\left[\left(1+2 u^{T} z+z^{T} z\right)^{2}-1 /\left(1+2 u^{T} z+z^{T} z\right)\right]
\end{aligned}
$$

\subsection{Invariants of the Right Cauchy-Green Deformation Tensor}

The thickness of the element in the deformed state can be removed from the expressions of the invariants of the right Cauchy-Green deformation tensor $C$. The invariants of the right Cauchy-Green deformation tensor and its derivatives with respect to the nodal displacements can be written as:

$$
\begin{gathered}
c_{1}=1+2 u^{T} z+z^{T} z+2 /\left(1+2 u^{T} z+z^{T} z\right)^{1 / 2} \\
\frac{\partial c_{1}}{\partial d^{1}}=-\frac{2}{\lambda}\left[1-\left(1+2 u^{T} z+z^{T} z\right)^{-\frac{3}{2}}\right](u+z) \\
\frac{\partial c_{1}}{\partial d^{2}}=+\frac{2}{\lambda}\left[1-\left(1+2 u^{T} z+z^{T} z\right)^{-\frac{3}{2}}\right](u+z) \\
c_{2}=\left(1+2 u^{T} z+z^{T} z\right)^{2}+2 /\left(1+2 u^{T} z+z^{T} z\right) \\
\frac{\partial c_{2}}{\partial d^{1}}=-\frac{4}{\lambda}\left[1+2 u^{T} z+z^{T} z-\left(1+2 u^{T} z+z^{T} z\right)^{-2}\right](u+z) \\
\frac{\partial c_{2}}{\partial d^{1}}=-\frac{4}{\lambda}\left[1+2 u^{T} z+z^{T} z-\left(1+2 u^{T} z+z^{T} z\right)^{-2}\right](u+z)
\end{gathered}
$$

\section{UNCONSTRAINED NONLINEAR PROGRAMMING PROBLEM}

A computer code was implemented following algorithms described by (Gill and Murray, 1974) and (Nocedal and Wright, 2006). It uses the limited memory BFGS quasi-Newton method in order to tackle large scale problems. The line search procedure is accomplished through cubic interpolation. The algorithm will find a local minimum point or in rare cases a saddle point. It can be easily added in the computer program implementation, the possibility of differentiating a local minimum point from a saddle point, and in the latter case proceed in the search for a local minimum using a direction of negative curvature. The minimization of the total potential energy approach to find equilibrium does not require preventing rigid body motion for self-equilibrated loading. This approach to find equilibrium can also replace the dynamic relaxation method as used by (Grancicova and Brodniansky, 2015). The source code written in Ada is available for download from www.arcaro.org. The script file for AutoCAD generated by the computer code allows the visualization of the undeformed and deformed shapes.

\section{EXAMPLES}

The blue color represents a strut element under tension while the red color represents a strut element under compression. The strut ele- 
ments have area equal to 1 . The examples consider an incompressible Mooney-Rivlin strain energy density function for a rubber-like material. The strain energy density function for this material is given by:

$$
\begin{aligned}
& \psi=\mu_{10} c_{1}+\left(c_{1}^{2}-c_{2}\right) \mu_{01} / 2 \\
& \mu_{10}=+0.375 \\
& \mu_{01}=-0.125
\end{aligned}
$$

The examples were compared with the ABAQUS finite element analysis software (Dassault Systemes, 2016), showing the maximum relative error for node displacement and for element stress. The displacement of each node was considered as a vector and the Euclidean norm was used to measure the relative error for vectors. ABAQUS uses the following expression for the strain energy density function of incompressible Mooney-Rivlin material, where $\kappa$ is the penalty parameter to enforce incompressibility.

$$
\psi=\mu_{10} c_{1}+\left(c_{1}^{2}-c_{2}\right) \mu_{01} / 2+\left(f_{3}-1\right)^{2} \kappa / 2
$$

The parameters used in the limited memory BFGS with a line search procedure through cubic interpolation are: (1) The maximum number of iterations allowed is usually 10 times the number of unknowns. (2) The maximum value allowed for the infinity norm of the gradient. The iterations terminate if the infinity norm of the gradient becomes less than or equal to this value. (3) The maximum number of cubic interpolations allowed is usually 20. (4) The accuracy of the line search is usually 0.1 . (5) The number of BFGS corrections kept is usually between 3 and 20 .

Example 1: The structure is defined by 4 nodes and 6 strut elements in the shape of a tetrahedron inscribed in a sphere of radius equal to 1 . The basis of the tetrahedron is parallel to the xy-plane. The origin of the coordinate system is located at the geometric centroid of the nodes. Table 1 shows the node coordinates and the zero displacement constraints to prevent rigid body movements. The parameters for the node coordinates are given by the following expressions.

$$
\eta_{1}=(2 / 3)^{1 / 2}, \eta_{2}=2^{1 / 2} / 3, \eta_{3}=2^{3 / 2} / 3, \eta_{4}=1 / 3
$$

Tab. 1 Node coordinates and displacement constraints

\begin{tabular}{|c|c|c|c|c|}
\hline node & $\mathrm{x}$ & $\mathrm{y}$ & $\mathrm{z}$ & constraints \\
\hline 1 & $-\eta_{1}$ & $-\eta_{2}$ & $-\eta_{4}$ & $\mathrm{z}$ \\
\hline 2 & $\eta_{1}$ & $-\eta_{2}$ & $-\eta_{4}$ & $\mathrm{z}$ \\
\hline 3 & 0 & $\eta_{3}$ & $-\eta_{4}$ & $\mathrm{x}, \mathrm{z}$ \\
\hline 4 & 0 & 0 & 1 & $\mathrm{x}, \mathrm{y}$ \\
\hline
\end{tabular}

Each node has an applied force equal to the node's coordinates. This loading will make the tetrahedron expands away from its geometric centroid. Figure 3 shows the initial and final shapes.
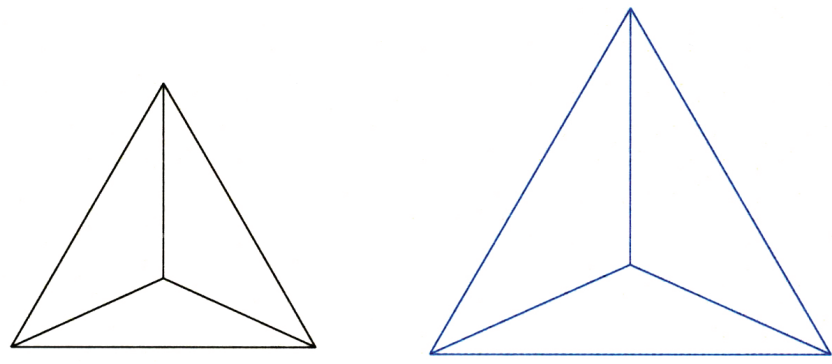

Table 2 shows the node displacement and the element stress that resulted in the maximum relative errors of 2.5E-06 and 2.6E-06 respectively when compared with ABAQUS.

Tab. 2 Node displacement and element stress

\begin{tabular}{|c|c|c|c|}
\hline Node & Displ X & Displ Y & Displ Z \\
\hline 2 & $2.542243 \mathrm{E}-01$ & $-1.467765 \mathrm{E}-01$ & $0.000000 \mathrm{E}+00$ \\
\hline Elem & Node & Node & Stress \\
\hline 1 & 1 & 2 & $5.353604 \mathrm{E}-01$ \\
\hline
\end{tabular}

Example 2: The structure is defined by 8 nodes and 12 strut elements in the shape of a hexahedron inscribed in a sphere of radius equal to 1 . The basis of the hexahedron is parallel to the xy-plane. The origin of the coordinate system is located at the geometric centroid of the nodes. Table 3 shows the node coordinates and the zero displacement constraints to prevent rigid body movements. The parameters for the node coordinates are given by the following expressions.

$$
\eta_{1}=(2 / 3)^{1 / 2}, \eta_{2}=1 / 3^{1 / 2}
$$

Tab. 3 Node coordinates and displacement constraints

\begin{tabular}{|c|c|c|c|c|}
\hline node & $\mathrm{x}$ & $\mathrm{y}$ & $\mathrm{z}$ & constraints \\
\hline 1 & $-\eta_{1}$ & 0 & $-\eta_{2}$ & $\mathrm{y}, \mathrm{z}$ \\
\hline 2 & 0 & $-\eta_{1}$ & $-\eta_{2}$ & $\mathrm{x}, \mathrm{z}$ \\
\hline 3 & $\eta_{1}$ & 0 & $-\eta_{2}$ & $\mathrm{y}, \mathrm{z}$ \\
\hline 4 & 0 & $\eta_{1}$ & $-\eta_{2}$ & $\mathrm{x}, \mathrm{z}$ \\
\hline 5 & $-\eta_{1}$ & 0 & $\eta_{2}$ & $\mathrm{y}$ \\
\hline 6 & 0 & $-\eta_{1}$ & $\eta_{2}$ & $\mathrm{x}$ \\
\hline 7 & $\eta_{1}$ & 0 & $\eta_{2}$ & $\mathrm{y}$ \\
\hline 8 & 0 & $\eta_{1}$ & $\eta_{2}$ & $\mathrm{x}$ \\
\hline
\end{tabular}

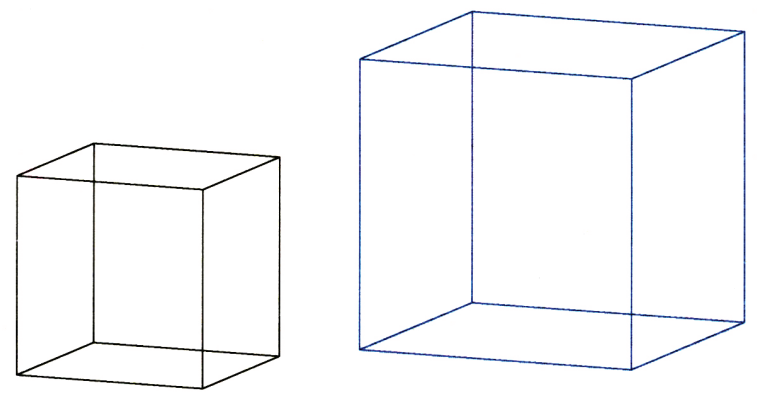

Fig. 4 Initial and final shapes

Each node has an applied force equal to the node's coordinates. This loading will make the hexahedron expands away from its geometric centroid. Figure 4 shows the initial and final shapes.

Table 4 shows the node displacement and the element stress that resulted in the maximum relative errors of 4.6E-06 and 5.6E-06 respectively when compared with ABAQUS.

Fig. 3 Initial and final shapes 
Tab. 4 Node displacement and element stress

\begin{tabular}{|c|c|c|c|}
\hline Node & Displ X & Displ Y & Displ Z \\
\hline 8 & $0.000000 \mathrm{E}+00$ & $3.785512 \mathrm{E}-01$ & $5.353523 \mathrm{E}-01$ \\
\hline Elem & Node & Node & Stress \\
\hline 1 & 1 & 2 & $8.450264 \mathrm{E}-01$ \\
\hline
\end{tabular}

Example 3: The structure is defined by 6 nodes and 12 strut elements in the shape of an octahedron inscribed in a sphere of radius equal to 1 . The origin of the coordinate system is located at the geometric centroid of the nodes. There are no constraints to prevent rigid body movements. Table 5 shows the node coordinates and, for ABAQUS, the zero displacement constraints to prevent rigid body movements.

Tab. 5 Node coordinates and displacement constraints

\begin{tabular}{|c|c|c|c|c|}
\hline node & $\mathrm{x}$ & $\mathrm{y}$ & $\mathrm{z}$ & constraints \\
\hline 1 & -1 & 0 & 0 & $\mathrm{y}, \mathrm{z}$ \\
\hline 2 & 0 & -1 & 0 & $\mathrm{x}, \mathrm{z}$ \\
\hline 3 & 1 & 0 & 0 & $\mathrm{y}, \mathrm{z}$ \\
\hline 4 & 0 & 1 & 0 & $\mathrm{x}, \mathrm{z}$ \\
\hline 5 & 0 & 0 & -1 & $\mathrm{x}, \mathrm{y}$ \\
\hline 6 & 0 & 0 & 1 & $\mathrm{x}, \mathrm{y}$ \\
\hline
\end{tabular}

Each node has an applied force equal to the node's coordinates. This loading will make the octahedron expands away from its geometric centroid. Figure 5 shows the initial and final shapes.
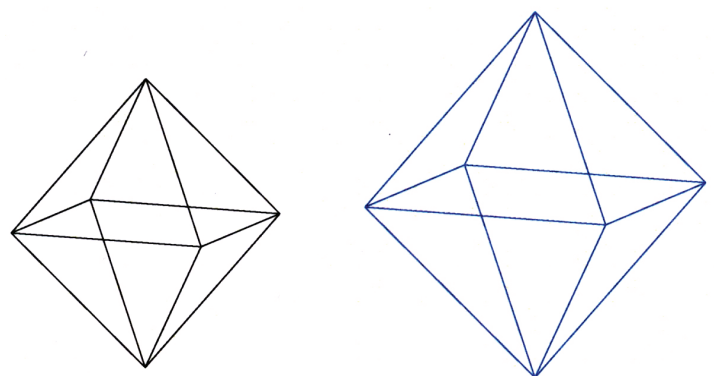

Fig. 5 Initial and final shapes

Table 6 shows the node displacement and the element stress that resulted in the maximum relative errors of $1.5 \mathrm{E}-06$ and $1.8 \mathrm{E}-06$ respectively when compared with ABAQUS.

Tab. 6 Node displacement and element stress

\begin{tabular}{|c|c|c|c|}
\hline Node & Displ X & Displ Y & Displ Z \\
\hline 6 & $0.000000 \mathrm{E}+00$ & $0.000000 \mathrm{E}+00$ & $2.649892 \mathrm{E}-01$ \\
\hline Elem & Node & Node & Stress \\
\hline 1 & 1 & 2 & $4.472412 \mathrm{E}-01$ \\
\hline
\end{tabular}

Example 4: The structure is defined by 20 nodes and 30 strut elements in the shape of a dodecahedron inscribed in a sphere of radius equal to 1 . The origin of the coordinate system is located at the geometric centroid of the nodes. There are no constraints to prevent rigid body movements. Table 7 shows the node coordinates and, for ABAQUS, the zero displacement constraints to prevent rigid body movements. The parameters for the node coordinates are given by the following expressions.

$$
\eta_{1}=\left(1+5^{1 / 2}\right) /\left(3^{1 / 2} 2\right), \eta_{2}=2 /\left[\left(1+5^{1 / 2}\right) 3^{1 / 2}\right], \eta_{3}=1 / 3^{1 / 2}
$$

Tab. 7 Node coordinates and displacement constraints

\begin{tabular}{|c|c|c|c|c|}
\hline node & $\mathrm{x}$ & $\mathrm{y}$ & $\mathrm{z}$ & constraints \\
\hline 1 & 0 & $\eta_{2}$ & $\eta_{1}$ & $\mathrm{x}$ \\
\hline 2 & 0 & $-\eta_{2}$ & $\eta_{1}$ & $\mathrm{x}$ \\
\hline 3 & $\eta_{3}$ & $\eta_{3}$ & $\eta_{3}$ & \\
\hline 4 & $-\eta_{3}$ & $\eta_{3}$ & $\eta_{3}$ & \\
\hline 5 & $-\eta_{3}$ & $-\eta_{3}$ & $\eta_{3}$ & \\
\hline 6 & $\eta_{3}$ & $-\eta_{3}$ & $\eta_{3}$ & \\
\hline 7 & $\eta_{1}$ & 0 & $\eta_{2}$ & $\mathrm{y}$ \\
\hline 8 & $-\eta_{1}$ & 0 & $\eta_{2}$ & $\mathrm{y}$ \\
\hline 9 & $\eta_{2}$ & $\eta_{1}$ & 0 & $\mathrm{z}$ \\
\hline 10 & $-\eta_{2}$ & $\eta_{1}$ & 0 & z \\
\hline 11 & $-\eta_{2}$ & $-\eta_{1}$ & 0 & z \\
\hline 12 & $-\eta_{2}$ & $-\eta_{1}$ & 0 & z \\
\hline 13 & $\eta_{1}$ & 0 & $-\eta_{2}$ & $\mathrm{y}$ \\
\hline 14 & $-\eta_{1}$ & 0 & $-\eta_{2}$ & $\mathrm{y}$ \\
\hline 15 & $\eta_{3}$ & $\eta_{3}$ & $-\eta_{3}$ & \\
\hline 16 & $-\eta_{3}$ & $\eta_{3}$ & $-\eta_{3}$ & \\
\hline 17 & $-\eta_{3}$ & $-\eta_{3}$ & $-\eta_{3}$ & \\
\hline 18 & $\eta_{3}$ & $-\eta_{3}$ & $\eta_{3}$ & \\
\hline 19 & 0 & $\eta_{2}$ & $-\eta_{1}$ & $\mathrm{x}$ \\
\hline 20 & 0 & $-\eta_{2}$ & $-\eta_{1}$ & $\mathrm{x}$ \\
\hline
\end{tabular}

Each node has an applied force equal to the node's coordinates. This loading will make the dodecahedron expands away from its geometric centroid. Figure 6 shows the initial and final shapes. ABAQUS was not able to run this example.

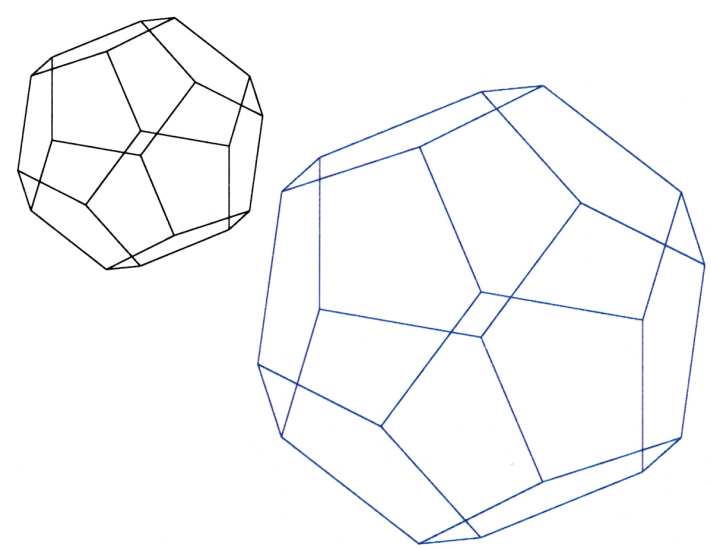

Fig. 6 Initial and final shapes 
Example 5: The structure is defined by 12 nodes and 30 strut elements in the shape of an icosahedron inscribed in a sphere of radius equal to 1 . The origin of the coordinate system is located at the geometric centroid of the nodes. There are no constraints to prevent rigid body movements. Table 8 shows the node coordinates and, for ABAQUS, the zero displacement constraints to prevent rigid body movements. The parameters for the node coordinates are given by the following expressions.

$$
\eta_{1}=\left[2 /\left(5^{1 / 2}+5\right)\right]^{1 / 2}, \eta_{2}=\left[\left(5^{1 / 2}+5\right) / 10\right]^{1 / 2}
$$

Tab. 8 Node coordinates and displacement constraints

\begin{tabular}{|c|c|c|c|c|}
\hline node & $\mathrm{x}$ & $\mathrm{y}$ & $\mathrm{z}$ & constraints \\
\hline 1 & $\eta_{1}$ & 0 & $\eta_{2}$ & $\mathrm{y}$ \\
\hline 2 & $-\eta_{1}$ & 0 & $\eta_{2}$ & $\mathrm{y}$ \\
\hline 3 & 0 & $\eta_{2}$ & $\eta_{1}$ & $\mathrm{x}$ \\
\hline 4 & 0 & $-\eta_{2}$ & $\eta_{1}$ & $\mathrm{x}$ \\
\hline 5 & $\eta_{2}$ & $\eta_{1}$ & 0 & $\mathrm{z}$ \\
\hline 6 & $-\eta_{2}$ & $\eta_{1}$ & 0 & $\mathrm{z}$ \\
\hline 7 & $-\eta_{2}$ & $-\eta_{1}$ & 0 & $\mathrm{z}$ \\
\hline 8 & $\eta_{2}$ & $\eta_{1}$ & 0 & $\mathrm{z}$ \\
\hline 9 & 0 & $\eta_{2}$ & $-\eta_{1}$ & $\mathrm{x}$ \\
\hline 10 & 0 & $-\eta_{2}$ & $-\eta_{1}$ & $\mathrm{x}$ \\
\hline 11 & $\eta_{1}$ & 0 & $-\eta_{2}$ & $\mathrm{y}$ \\
\hline 12 & $-\eta_{1}$ & 0 & $-\eta_{2}$ & $\mathrm{y}$ \\
\hline
\end{tabular}

There are two loadings: (1) each node has an applied force equal to the node's coordinates and (2) each node has an applied force equal to the negative node's coordinates. These loadings will make the icosahedron expands or shrinks away from its geometric centroid. Figure 7 shows the initial shape on the left and the final shapes on the right.

The structure expands. Table 9 shows the node displacement and the element stress that resulted in the maximum relative errors of 2.0E-06 and 2.2E-06 respectively when compared with ABAQUS.

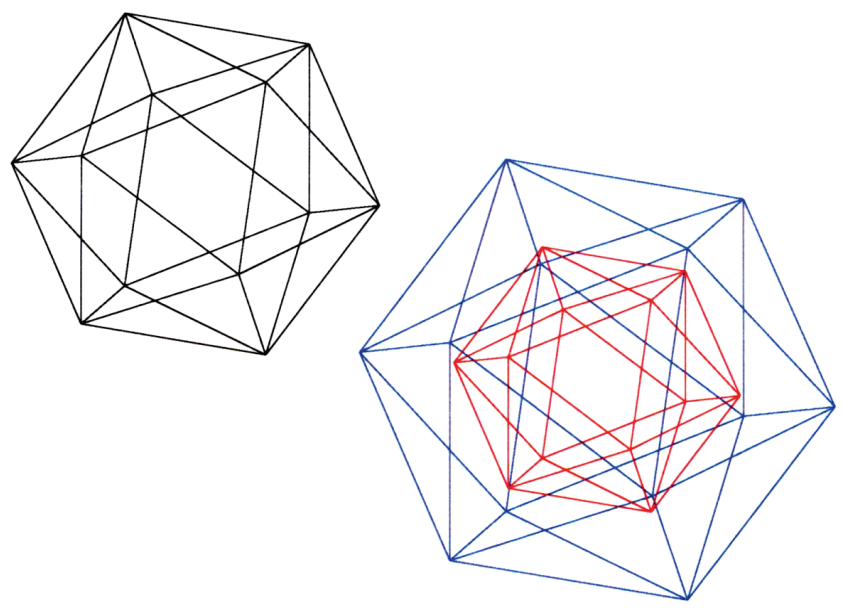

Fig. 7 Initial and final shapes
Tab. 9 Node displacement and element stress

\begin{tabular}{|c|c|c|c|}
\hline Node & Displ X & Displ Y & Displ Z \\
\hline 12 & $-1.511947 \mathrm{E}-01$ & $0.000000 \mathrm{E}+00$ & $-2.446382 \mathrm{E}-01$ \\
\hline Elem & Node & Node & Stress \\
\hline 1 & 1 & 2 & $4.898281 \mathrm{E}-01$ \\
\hline
\end{tabular}

The structure shrinks. Table 10 shows the node displacement and the element stress that resulted in the maximum relative errors of $1.6 \mathrm{E}-06$ and $1.4 \mathrm{E}-06$ respectively when compared with ABAQUS.

Tab. 10 Node displacement and element stress

\begin{tabular}{|c|c|c|c|}
\hline Node & Displ X & Displ Y & Displ Z \\
\hline 1 & $-1.183029 \mathrm{E}-01$ & $0.000000 \mathrm{E}+00$ & $-1.914181 \mathrm{E}-01$ \\
\hline Elem & Node & Node & Stress \\
\hline 1 & 1 & 2 & $-2.948178 \mathrm{E}-01$ \\
\hline
\end{tabular}

Example 6: The structure is defined by 468 nodes and 1332 strut elements in the shape of a cylinder with diameter equal to 1 and height equal to 1 . The cylinder was divided into 36 equal parts along its circumference and into 12 equal parts along its height. The origin of the coordinate system is located at the geometric centroid of the nodes. The displacements for the nodes located at the top and bottom circumferences were imposed equal to 0 . Each node has an applied force equal to the node's coordinates. This loading will make the cylinder expands away from its geometric centroid. The cylinder expands symmetrically while keeping the nodes located at the top and bottom circumferences fixed. Figure 8 shows the initial and final shapes.
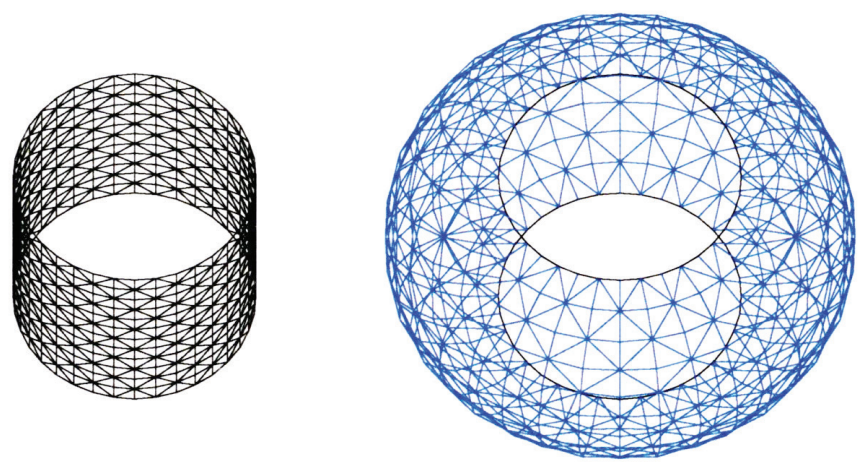

Fig. 8 Initial and final shapes

Table 11 shows the node displacement and the element stress that resulted in the maximum relative errors of 4.0E-06 and 1.4E-05 respectively when compared with ABAQUS.

Tab. 11 Node displacement and element stress

\begin{tabular}{|c|c|c|c|}
\hline Node & Displ X & Displ Y & Displ Z \\
\hline 208 & $-3.383873 E-14$ & $-4.549997 \mathrm{E}-01$ & $-4.748185 \mathrm{E}-02$ \\
\hline Elem & Node & Node & Stress \\
\hline 470 & 37 & 73 & $1.044470 \mathrm{E}+00$ \\
\hline
\end{tabular}


Example 7: The structure is defined by 468 nodes and 900 strut elements in the shape of a cylinder with diameter equal to 1 and height equal to 1 . The cylinder was divided into 36 equal parts along its circumference and into 12 equal parts along its height. The origin of the coordinate system is located at the geometric centroid of the nodes. There are no constraints to prevent rigid body movements. For ABAQUS, the nodes along the yz plane can not move on the $\mathrm{x}$ direction, the nodes along the $\mathrm{xz}$ plane can not move on the $\mathrm{y}$ direction, and the nodes along the middle circumference can not move along the $\mathrm{z}$ direction. Each node located at the top and bottom circumferences has an applied force equal to the node's coordinates. This loading will make the top and bottom circumferences expand away from the cylinder's geometric centroid. The final surface is symmetrical about the z-axis. Figure 9 shows the initial and final shapes. ABAQUS was not able to run this example.
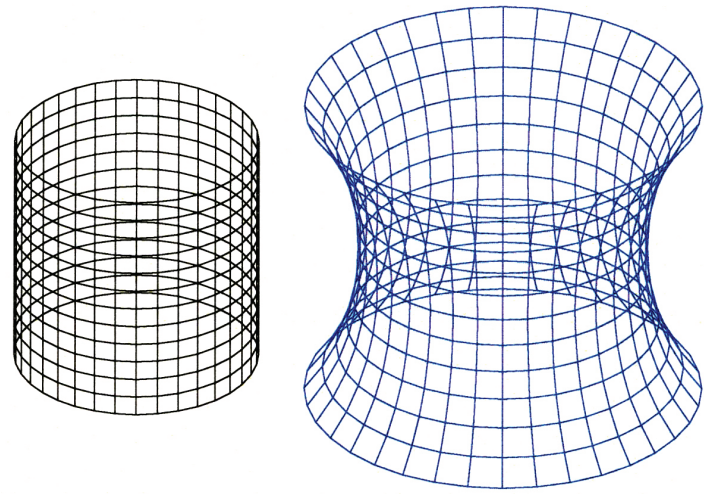

Fig. 9 Initial and final shapes

\section{Conclusions}

The results of the examples calculated by the proposed mathematical modeling show five significant digits in agreement when compared with the ABAQUS FEA software. The advantages of using a quasi-Newton method to minimize the total potential energy are: It is not necessary to derive the stiffness matrix expressions; it is not necessary to solve any system of equations; it does not require preventing rigid body motion for self-equilibrated loading. The source and executable computer codes are available for download from http://www. arcaro.org/. The computer code generates a script file for AutoCAD. 


\section{REFERENCES}

Buchholdt H. A. (1966) Deformation of prestressed cable-nets. Norwegian Academy of Technological Sciences, Norway.

Coyette J. P. - Guisset P. (1988) Cable network analysis by a nonlinear programming technique. Eng Struct 10:41-46.

Brinkhues S. - Klawonn A. - Rheinbach O. - Schroder J. (2013) Augmented Lagrange methods for quasi-incompressible materials-Applications to soft biological tissue. Int J Numer Meth Bio 29:332-350. doi: 10.1002/cnm.2504.

Hadjicharalambous M. - Lee J. - Smith N. P. - Nordsletten D. A. (2014) A displacement-based finite element formulation for incompressible and nearly-incompressible cardiac mechanics. Comput Method Appl M 274:213-236. doi: 10.1016/j. cma.2014.02.009.

Mustafy T. - El-Rich M. - Mesfar W. - Moglo K .(2014) Investigation of impact loading rate effects on the ligamentous cervical spinal load-partitioning using finite element model of functional spinal unit C2-C3. J Biomech 47:2891-2903. doi: 10.1016/j.jbiomech.2014.07.016.

Wex C. - Arndt S. - Stoll A. - Bruns C. - Kupriyanova Y. (2015) Isotropic incompressible hyperelastic models for modelling the mechanical behaviour of biological tissues: a review. Biomed Tech (Berl) 60:577-592. doi: 10.1515/bmt-2014-0146.
Goldberg D. (1991) What every computer scientist should know about floating-point arithmetic. ACM Comput Surv 23:5-48. doi: 10.1145/103162.103163.

Kaklamanis C. A. - Spiliopoulos K. V. (2007) A general formulation for large strains hyperelastic trusses. 8th HSTAM International Congress on Mechanics, Patras, Greece.

Bonet J. - Wood R. D. (2008) Nonlinear continuum mechanics for finite element analysis, 2nd edition. Cambridge University Press.

Gill P. E. - Murray W. (1974) Newton type methods for unconstrained and linearly constrained optimization. Math Program 7:311-350. doi: 10.1007/BF01585529.

Nocedal J. - Wright S. J. (2006) Numerical optimization, 2nd edition. Springer-Verlag

Grancicova I. - Brodniansky J. (2015) Estimation of Young's modulus of elasticity by the form finding of grid shell structures by the dynamic relaxation method. Slovak Journal of Civil Engineering 23:25-30. doi: 10.1515/sjce-2015-0020.

ABAQUS, Dassault Systemes, (2016) Velizy-Villacoublay, France. 